\title{
Software Project Work in an African Context: Myths, Maps and Messes
}

\author{
Muhammad Sadi Adamu \\ School of Computing and Communications, Lancaster University, UK \\ m.adamu@lancaster.ac.uk
}

\begin{abstract}
Research in HCI and CSCW has consistently shown how software design approaches are an abstract idealisation of work practices, raising questions regarding the appropriateness and applicability of what might be considered as 'best practice' or 'doable practice' in project work. Such issues have magnified the fundamental need for examining exactly how conventional (and generally Western) constructs, approaches and methods, widely adopted in the process of producing and deploying technologies, actually work. The paper reports findings from a study that seeks to understand the implications for adopting 'well-known' practices for framing, undertaking, and analysis distributed and collaborative software project in the context of Nigeria. Findings show that documenting and analysing what is often considered as 'best practice', supposedly prescriptive maps and scripts for accomplishing work, necessitates considering how they get adopted, interpreted, and extended as 'orderly' and occasionally 'messy' alternatives, offering some sensitivities for understanding the translocal features and transitional meaning of agile project work.
\end{abstract}

\section{CCS CONCEPTS}

- Human-centered computing $\rightarrow$ Human computer interaction (HCI); Empirical studies in HCI.

\section{KEYWORDS}

Africa, Software Project, Transitionality, Translocality, Agility, Culture, Context

ACM Reference Format:

Muhammad Sadi Adamu. 2020. Software Project Work in an African Context: Myths, Maps and Messes. In 32nd Australian Conference on Human-Computer Interaction (OzCHI '20), December 02-04, 2020, Sydney, NSW, Australia. ACM, New York, NY, USA, 14 pages. https://doi.org/10.1145/3441000.3441071

\section{INTRODUCTION}

In contemporary society, there is a general assumption that technology can and will revolutionize the way we live, think and act. However, research in $\mathrm{HCI}$ and ubiquitous computing has shown how conventional approaches to understanding cultures and values

Permission to make digital or hard copies of all or part of this work for personal or classroom use is granted without fee provided that copies are not made or distributed for profit or commercial advantage and that copies bear this notice and the full citation on the first page. Copyrights for components of this work owned by others than the author(s) must be honored. Abstracting with credit is permitted. To copy otherwise, or republish, to post on servers or to redistribute to lists, requires prior specific permission and/or a fee. Request permissions from permissions@acm.org.

OzCHI '20, December 02-04, 2020, Sydney, NSW, Australia

(c) 2020 Copyright held by the owner/author(s). Publication rights licensed to ACM ACM ISBN 978-1-4503-8975-4/20/12 .. \$15.00

https://doi.org/10.1145/3441000.3441071 are developed in relation to and within modernistic frames that determine what is relevant and what is not - mostly Eurocentric [11]. This raises a range of questions concerning how certain methodological and analytical practices get privileged, monopolized, and normalized. And of what that might mean and suggest concerning 'best practices' - best for who? from where? for what purpose? and at what cost? This, therefore, presents any approach for framing project work to be an asymmetric relation that needs to be critically and continuously appropriated $[6,34]$. This is important as it allows an understanding of how dominant conventions (both technological and socio-cultural) influence the practice of designing and deploying technological innovation in Africa.

Even with the continual call for the inversion of design paradigms and lenses in $\mathrm{HCI}$ and $\mathrm{CSCW}$, few studies from Nigeria have examined the design and development frames informing the work of software practitioners [24,26-28]. What these studies have shown are the assumptions and principles shaping the practice of the community; specifically, the (mis)understanding of 'user's', 'cultures' and 'politics' in design and the approaches adopted and used for designing, developing and evaluating tools. From these studies, it becomes apparent that most of the approaches adopted are Eurocentric and effectively neo-colonial. This might, therefore, present the adoption and integration a range of approaches (as prescriptive maps and scripts [32]) in the everyday work of software practitioner as an expensive gamble due to the differences in the culture of initiation and the context of appropriation. Research has not established how well-known approaches, specifically agile methodology and a range of design methods, actually work in a multicultural context such as Nigeria. This case study examines how a range of design and methodological practices get applied, contested, ignored, and extended in distributed and collaborative project work. This would show how certain organisational practices are monopolised, how software development methodologies are universalised, how design approaches are conventionalised, and how management knowledge is totalised through a globalist matrix of power relations.

To examine the matrix of power relations in the practice of project work, I draw on the analytical and cultural approach of 'translocality' in sensitizing and evaluating the mundane practice of software project work in three Nigerian software companies referred to as Edusoft projects [6]. Using qualitative data, the case study seeks to answer the question: How does the Edusoft projects do agility under the influence of civic structures and organisational contingencies in the overall practice of work? The study identifies and documents the implications of adopting and using well-known approaches for framing, undertaking and analysing distributed and collaborative software project work. This challenges the basic assumption that software practitioners in/from Africa are merely recipients of transfer, imitators of Western innovation, or victims 
of transplantation and appropriation [22, 40]; instead showing how they continuously innovate new practices that get distributed across boundaries.

Adding to the important commitment of deconstructing Western approaches to technology design and development, the case study makes a range of contributions. First, empirical evidence on the situated nature of doing agility in software project work is presented. Using the case of three companies in Nigeria as a perspicuous example, the six themes identified suggest how the adoption of conventions (or lack of them) and the standardization of work have led to a range of developmental difficulties, poorly reflect the context of use, might lead to the likelihood of project failing, or might even warrant less acceptance and use of deployed tools [6, 37]. Second, the case reiterates the conceptual implications of 'transitionality' and 'translocality' in decoding and deconstructing the politics and materiality of project work across contested boundaries [6, 12]. Transitionality is a critical approach to design that is driven by the praxis of border thinking and making for the pluriverse [12]. Translocality on the other hand provides a way of showing the agility of Edusoft projects as a localised circumstance that is emerging in everyday practice [6]. This suggests that the issues of scaling design approaches across boundaries are not merely about numbers but about the continual shifting of relation across multiple cultural context [4]. This places the need for examining how local experiences can offer a way of scaling developmental methodologies and analytical approaches without reinstating the binaries of the globallocal. Third, the case also indicates the methodological implication of 'remixing' and 'playfulness' in regenerating and redistributing work practices, which might show the plurality of design practices and how they communicate across cultures. Finally, the case might also be considered as restating and reformulating Suchman's seminal arguments about the contingency of rules and rule-following as applied to non-Western software practices, which thereby highlight the 'mess' rules might create as a consequence of the inevitable situated nature of work, which, in turn, perhaps necessitates new views of the notion of scale across contested relations and emerging boundaries.

\section{BACKGROUND}

In the global South, the research of information system (IS) has gained significant attention due to the widening of the technological divide, the failure of technology adoption and diffusion across boundaries, and of the oversimplification of the potentials of technology to socio-cultural and economic development $[3,15]$. Research in postcolonial and indigenous $\mathrm{HCI}$ has also pointed to the need for examining the practices of innovation as a transnational issue where socio-cultural, economic, political, and material relations are made more open for both analysis and regeneration $[1,17,34,35]$. Transnational HCI and its associated approaches to understanding culture and context are considered as analytical lenses of examining the shifting associations between the global and the local [34]. What transnationality offers is an approach for articulating and contextualization alternatives practices of global software development. This, therefore, calls for an examination of how localised practices unfold as they get incorporated and absorbed in the exchange of and encounter with new challenges and opportunities.

Besides, there has also been a greater interest in research that moves away from the approaches to the design and development of innovation to understanding how they enacted in the actuality of project work, and the gap and mismatch that such account creates $[19,24,25,39]$. What these studies have shown is the complex nature and contested aspect of doing design work in a world characterised by the global-local, in here-out there, centre-margin. There is also the continual debate about the relevance of 'well-known' design approaches and their link to imperialist traditions of classification, hierarchization and misrepresentation [35]. The widely held assumption is that conventions are expected to guide and direct passive recipients, ignoring alternate reasonings of transitional design $[33,38]$. In this paper, I approach these issues by reflexively analysing how what is often considered as 'best practices' are constitutively appropriated and regenerated across contested and existing structures of global agile.

Consequently, the agile approach came as an iterative, adaptive, flexible, rapid and evolving ways of doing software project work. Regardless of its potentials and prospect to undertaken and accomplishing work, research has made clear how it has been used and misused in a range of social context - either as a mode of standardization or as a sensitivity that encourages temporality and flexibility $[5,10,20,25,37,39]$. There is also the question of whether the methodologies adopted represent actual work of practitioners or whether it is some idealized form of project work (or whether agility makes work messy)? [10]; whether the use of agile methodology actually does agility, and in some way improve the processes and activities of software practitioners? [18]; whether agility is some idealized notion of an adaptive and responsive methodology that makes work unnecessarily difficult and more complicated than it ought to be? [3,5]. Findings point to the relevance and limitation of agility in transnational spaces and of how other operational variables and feature determine its (in)effectiveness in various circumstances. The case presented in this paper examines the socio-cultural and contextual variables that inform and shape the agility of Edusoft projects. This might signal the utility of conventional approach to work in $\mathrm{CSCW}$, as much as design methods in $\mathrm{HCI}$, while also identifying the resourcefulness of local logicalities of appropriation and usage.

\section{EMPIRICAL STUDY}

\subsection{Settings and Methods}

The case study is part of an interdisciplinary research project that seeks to decode and deconstruct approaches to understanding and designing educational technologies to be adopted and used by a range of stakeholders in Nigerian universities. Nigeria is widely considered as the giant of Africa, its powerhouse, its largest economy, and surprisingly, the poverty capital of Africa. The software industry in Nigeria has seen some considerable patronage partly because both the public and private sector have championed for local content development, acquisition, and promotion. Policies and strategic actions plans are often outlined by political governments, however, the issue that hinders the needed development of the 
industry are the lack of adequate implementation of strategies and the enforcement of policy initiatives. The practice of the industry can be best characterised has 'translocal', 'transient', and 'transversal'. However, partly due to the instability in government actions plans for the industry, and also partly but significantly due to the perception of the general public towards local vendor products, the industry has suffered from the lack of political commitment and public patronage.

The paper is informed by the consideration of how intersectional lenses can offer ways to delink from dominant assumptions and relink to indigenous perspectives within the framing of transnational design. The case considers the techniques, procedures, and technologies shaping the work of producing adaptable, usable and saleable educational tools in Nigeria. The emphasis here is not on theorizing work practices, but of identifying and discussing how stereotypical approaches get absorbed, interpreted, applied, and extended in multicultural collaborative work. This is important as it may account for how agility is carried out in an organisational context where the influence of civic structures and organisational contingencies is apparent. It also shows how the situated indigeneity of practitioners allows for a constitutive appropriation and regeneration of new challenges and opportunities for accomplishing project work. This, therefore, warrants a closer examination of what 'doing' agility entails in a multifaceted context such as Nigeria, and of whether the meaning one attaches to agility 'out there' is the same as the agility practices 'in here'. This is relevant to those working on multicultural projects as it outlines a range of ideas as to whether and how to diffuse, appropriate, and translate alternative ways of thinking and doing agility.

The study was carried out in three locally owned companies that offer educational systems and services to the Nigerian educational landscape - specifically to schools, tertiary institutions, private sector companies, and government agencies. The case draws on qualitative data collected during two fieldwork sessions (in July 2018 and May 2019). During the initial fieldwork, seven interviews were conducted with practitioners in the companies (two project managers F1/F5, three designers/developers F2/F4/F6, an eLearning analyst F3, and a system analyst F7). The companies are located in Abuja (C1, C2) and Lagos (C3). Working with a range of companies (which service different clients in the northern and the southern part of Nigeria) provides a broad understanding of software work practice in Nigeria. From the initial analysis of the interview data, I noticed conflicting accounts of work among participants. For example, some suggested adopting agile methodology, others said waterfall methodology, while another suggested the notion of "partial agile' (participants term to denote the lack of following the entire principles of agile in their work).

During the follow-up fieldwork, I engaged six participants (a project manager FF5, a designers FF4, two developers FF2/FF3, the engineering group lead FF6, and the eLearning lead FF1) in C1 to develop a deeper and more insightful account of how their project work is carried out. Out of the almost 50 staff in their office, five are managerial managers, five are part of the eLearning team, fifteen part of the engineering team, and twenty-five as part of the CRM team. Within the engineering team, five senior developers work remotely, which makes the work of Edusoft distributed and collaborative. I engaged in observations of work processes, made conversations here and there, took notes and pictures where possible, and discussed organisational documents (e.g., the objective and key result (OKR)). I attended daily stand-ups, a sprint meeting and the weekly mock-up; taking notes of how work was organised and negotiated using a range of techniques, strategies and technologies. Although a relatively 'rapid ethnography' [23], I kept a journal $\log$ s of field activities and for field notes. Due to implicating points raised by [29], considering a perspicuous setting of $\mathrm{C} 1$ and not all three-setting become necessary.

\subsection{Analytical Approaches}

For analysis, I adopted a grounded approach to thematic analysis [13]. This was achieved through the examination of the interview transcripts where common themes were identified (stepwise replication) and agreed upon by the author and an academic advisor's (intercoder agreement). For the ethnographic data (consisting of conversation transcripts, notes, picture and organisation document), a descriptive story was developed. The notion of temporal trajectory was employed to emphasise the findings from the two studies. However, it is not presumed that the mundane activities and processes between the companies are the same, but on the premise that similar and relevant part can give a picture of the whole when critically and sensibly analysed. The working back and forth between the initial themes and the descriptive stories might suggest the complexities of the experiences and perspectives of participants over time. As writing and presentation of ethnographic account is inevitably selective and shaped by the positionality of the narrator, I practised different forms of member checking of the interview transcript, reciprocal evaluation of themes and the validation of descriptive stories with participants. In the reportage and representation of participants work, I adopted the classic metaphor of synecdoche to generalise to the education software industry in Nigeria. However, I identify with [8] rhetorical construct and assertions, which warrants taking a generable stand across the Nigerian software industry. The findings might be different from other social context in Africa, as such not claiming to provide a representation of the African context with the case provided. As the focus of the paper is on sensitizing and evaluating the practices of software project work in Nigeria, it becomes necessary to document how Edusoft projects work is framed and undertaken.

\section{FINDINGS}

\subsection{The Orderliness and Messiness of Edusoft projects}

For a project that is distributed and collaborative, practitioners work together and sometimes against each other as to ensure that project works are kept on track. In this subsection, I examine the orderliness and occasional messiness of Edusoft projects to show how different approaches inform and shape project work. Apart from adopting agile methodologies in organising and ensuring projects are carried out through established practices, the C1's strategic approach for work was to order project work through phases of project initiation and assessment, design and development planning, project execution, and project management. But Edusoft projects are inevitably situated and shifting in nature; the adoption of certain procedures 
and technologies might support the orderliness of work (considered as maps and scripts for keeping work as a totality) or might, inadvertently, make work messy. Specific emphasis is placed on understanding how agility might create order/mess? How changes are affected when plans don't work out? How conflict is handled and absorbed in an attempt to keep work in totality? And of how localized logicalities (e.g., the use of OKR's) might have assisted in making those messy circumstances productive. Accounting for such instances shows the contingent effect of rules and their following as applied to different circumstances.

Project Initiation and Assessment: When a new project is initiated, be it based on user requirement or an in-house initiative, specific project deliverables are identified and generated. During project briefs, project goals, conceptual plans and derivatives are drafted. First, in the initiation and assessment phase, concepts detailing the entire project are examined. This includes the desirables and deliverables, timeline and phases, budget and resources, and the expected attributes of the project. This is a crucial phase of any project, where all processes and expected outcomes are outlined following the project goals and C1's mission. In company C1, the Jira agile board is used for assessing, planning, tracking and executing the entire processes of Edusoft projects. Jira is widely considered as a tool for the effective utilization of the agile methodology. As indicated in Figure 3 and Figure 4, Edusoft uses the Nemis scrum board for new projects, while Kanban is used for smaller maintenance projects. Other project management tools like Slack (for communication), Jenkins (for integrating codes and running crone job), bitbucket (for code version control), and Hugo.ia (for managing meetings) assist in ordering work. These tools are adopted to react to the requirement of active user engagement, distributed nature of work, and the need for keeping work orderly and as a totality. In this stage also, an analysis of the organizational processes is carried out to identify the resources and manpower needed for a particular project, while also outlining the different mechanism adopted in ensuring that the project is kept on track and within budgetary provisions.

System Design: In the planning, ideation and design phase, an indepth analysis of the different processes to be executed, and a clear itemizing of the different steps needed to translate the concepts outlined in the previous phase into a functional product are identified. As change is imminent and ambiguity is inevitable, Edusoft projects adopt a partial agile methodology. The partiality is partly due to the nature of projects, the influence of a range of contextual and organisational contingencies, the limitation of time on both clients and practitioners to fully articulate requirements, and the oversimplification of agility as a quick and dirty approach. For other smaller projects, Microsoft excel is used as a tool for ordering and managing planning activities. The use of excel was due to the nature of the project and the number of team members $(n=4-6)$. This might suggest that the nature of the project, the number of people working on the project, the uptake level and resources allocated for the project determines the tools to be used for planning and analysing requirements and design.

System Development and Evaluation: In the project execution stage, the design, development, and evaluation of technological artefacts are organized and managed on the Jira boards. The boards allow for the allocation of task to different people, which when completed and considered as a whole would produce a functioning product. How then does the use of Jira to undertake and accomplish work makes work orderly or does it make work more difficult and messier? [37]. What happens when plans don't work out? How do changes to plan get affected, get re-planned, negotiated and carried out in the design and development phase? [31]. Although agile calls for responding to change over following plans, what happens when changes keep coming, and the order of work becomes complicated and messy? Or does doing 'partial agile' provides a way of minimally following plans while also attempting to follow the agile guiding principles? From the analysis, it can be inferred that when changes occur at the organizational level, the departmental OKR's for the quarter are reviewed in line with the company OKR's and established practices in the industry. When changes are clientdriven, the project manager reviews client request with regards to how achievable and desirable they are - emphasizing collaboration over negotiation.

In addition, and more importantly, are the consequences of reacting to ever-changing requirements and how such flexibility might bring about new challenges to the ordering of software project (which might occasionally create a mess). The challenges mostly experienced in Edusoft projects are client-driven - i.e., clients pressuring practitioners to produce fully functional product within a shorter time frame. Participants pointed to different scenarios where changes are affected in projects, but the hierarchical relation remains the same - somewhat 'you are buying, we have to attend to your needs, we are selling, we have to leverage on maintaining profitability'. This, therefore, creates a conflicting scenario whereby they either stick to the plans outlined in the previous sprint (adding more workload to new sprints) or alter them as part of the agile need for responding to change (making work complicated and occasionally messy). Either way, rules following or being overly flexible complicated their everyday work. There is also the issue of how conflict or misunderstanding between team members are handled and absorbed in their work. For example, there appears to be a mismatch between what is 'ideal', 'what is doable', and what is 'good for business'. In such conflicting scenarios, team members are in constant communication and deliberation of optimal ways to attend to certain circumstances, in practical, democratic ways. The progression of project activities and processes are monitored real-time on the Jira board, which shows the transition of the engagement between different team members. The use of a range of tools and the individualisation of work has thus provided a way of not only focusing on the artefacts but the processes and the people involved.

System Deployment and Support: In the project management stage, the mechanism for determining whether the desired attributes of the project are achieved are employed through training and support provided to clients. It is in this stage that documentation is carried out as a mechanism for keeping a repository of project ideas and not some comprehensive reportage of the procedure followed in developing a functional system. What the analysis shows are the different procedures, strategies and technologies adopted in ensuring that Edusoft projects are ordered and kept on track and within budget and time frame. It also points to how localised indigeneities are translated in work to affect changes, minimise deviations, manage peer conflict, and keep project work on track. The 
different approaches adopted and used for Edusoft projects have indeed assisted in orienting work as a totality but have also created a possibly stressful and a difficult working environment - which might ultimately lead to a messier work culture [5, 37]. From the understanding of the mundane practices of Edusoft projects, there appears to be no 'this is the way', or 'this is the best way', software practitioners adopt, appropriate, adapt, and ignore different options with regards to how they make their work more productive and less difficult.

\subsection{Meta Themes}

Adding to how Edusoft adopts and adapt the agile methodology in their work, I identified six relational themes that emerged from the analysis of the interview transcript and ethnographic data gathered from practitioners in Nigeria. The themes outlined here are meant to account for the influence and impact of civic structures and organisational contingencies to the overall practice doing agility. The interpretation of such influences to the practices of Edusoft project would show how well-known constructs and concepts are experienced and expressed within the framing of translocal circumstances and perspectives - highlighting how local differences and difficulties identifies with adopting a largely Western imaginary of what design innovation and software development entails. The discussion of the themes would explicate the meanings attach to principles of the agile methodology, and how it assists in doing design innovation within the multicultural context of Africa, and specifically Nigerian.

4.2.1 Stakeholder Role's - Who Matters the Most?. This theme concerns the roles of different stakeholders in determining how software products get produced and used in Nigeria, specifically as they relate to requirement gathering, informing design, and the evaluation and acceptance of end product. From the analysis, the data suggests that 'administrators' - consisting of educational managers/technologies of a particular unit in institutions or organisation - are the people that matter the most in eliciting what and what's not of system requirement and acceptability. However, educational tools are developed and used by different users, e.g., those that teach, learn or manage the processes of teaching and learning, and those that manage the process of running educational enterprises. These users would have different interests, concerns, roles, positionality, and power dynamics. The rationale, as suggested by most participants was that 'stakeholders' are paying for the products and thus the more important persons when designing and developing educational products. In a participant's words;

"I think the most part is that we engage with the stakeholders (management) in gathering those requirements. ....If you are developing a product, you can go out and talk to people and gather some information from them or you can put yourself in the shoes of the user as no person is paying for it. But when someone is paying for such a product, they are actually the person that gives you the requirement" (EVF - FF2).

What this might suggest is that as educational tools are deployed for use by a range of users, the significance of the role of stakeholders would depend on the product to be developed. If the product is initiated based on some shared understanding of a particular problem (in-house), who matters the most would be the team member's working on the project (or in some instances the user group articulating the problem to be designed for). If it is a client-oriented project, the consideration of 'those paying' shows the political and economic relation of the role of stakeholder to the practice of software development. The emphasis is that due to the social situations (underdevelopment and economic hardship) and fragile nature of politics in Nigeria (which is about population and different factions), stakeholder's role matters when resources (people) are involved. A developer explained the implications of stakeholder's role in their everyday work by suggesting that;

\section{"With regard to gathering requirement and evaluation if the administrators would allow the actual users of the system to be the key subject, that would be more interesting. This is because we believe that engaging with the actual users will determine if we should be doing it in the first place or not. But the case here is that administrators do the saying and evaluation" (F6).}

Such ideas are generalizable to the Nigerian context, knowing the political atmosphere, and how socio-cultural affiliation influences the representation of people or shape the crisis of representing the perspectives of different people in design and development processes. The consideration of 'stakeholders' as the de-factor informant of project requirements might thus pose a range of developmental difficulties during design and development as work is driven by assumptions that are might not reflect end user's concerns and needs, thus presenting the greater chance of deployed tools not getting acceptance and adopted effectively.

4.2.2 Requirement Gathering and Analysis. This theme relates to ideas about how requirements are gathered and analysed for the design and development of educational technologies. Such ideas overlap with issues raised about the role of 'administrators' and 'end-users' in software projects, and what that might suggest to the situated practices of Edusoft projects. Findings suggest the use of classic methods of eliciting requirement (for example, interviews, questionnaires, online feedback forms, customer feedbacks and testimonies, product documentation, user research, system requirement specification documents). When a software product is based on some shared understanding of a particular problem, requirements are shaped by the practitioner's reflective recollection on the vital features and functionalities that a deployable tool ought to have. The project manager is responsible for explicitly identifying and analysing user requirements to the design and development team. Regardless of the technique adopted for gathering requirements, it is presumed that the Project manager has the experience and expertise to act as the system analyst, outlining the specifics of the project, conducting the feasibility analysis and making decisions either in consultation with other team members or alone. This is supported by four respondents admitting that they gather requirements from the 'administrators' and 'managers' of the institutions (F1, F3, F6, F7), while others believe that the ideal way is to speculatively 'think for the users of such tools' (F2, F4, F5) based on their understanding of vital user's needs and expectancies. For example, some participants suggested that; 
"Honestly, in most cases, these requirements don't necessarily reflect the perspective of the actual users.... we tend to guide users as to what might work or not" (F1)

"For that project, we spoke with *** (a government agency). There is no form of communication with the people that we are going to deploy for. Most times, when you deal with the government, they just tell you this is what we want. They don't allow this seamless process; they just have documented requirements. When we got those requirements, we believe the people that came up with the requirement are the educational and development team that have Moodle in mind. .....We concluded that the team in ${ }^{* * *}$ felt they were thinking like actual users, but they actually not. They just wanted a solution that will fit into the context of what they felt was right, which was not necessarily the right thing (F3)

"Our staff go to the field to gather requirement and talk to the university management and get their needs. ... Although the students are the actual users of the tools. The problem is that we are designing for the students, but a lot of times is what the administrator wants that is provided. It's always a challenge, to be honest.... Ideally, it should be the users that tell us what they want and assist us in evaluating it. But the case here is that the administrators do the saying and evaluation" (F6).

This might suggest a mismatch between those that determine how the products get designed, developed, and evaluated and those that get to use them. A project manager suggested that due to the socio-cultural conditions and political contingencies of the context practitioners work, stakeholder's role matters in most of the project phases mainly become their role has a political and economic implication. The implications were illustrated by the suggestion that;

"As a company, our primary focus is not on providing services but making some impact to the community. The government might not care much for value for money as there are a lot of political forces behind any government project. Due to the nature of the political atmosphere here, $80 \%$ of our projects are private sector-driven, while $20 \%$ government. In case there is any kind of instability in government policies, we are at least covered or will reduce the effect on the business" (FF5).

As Edusoft is a business enterprise saddled with social and cooperate responsibilities, practitioners have to be reactive to local situation and circumstances. The more prominent of which is that 'administrators' (both in government or the private sector) are the one's paying for the services provided, and any deviation or turbulence in the political system in Nigeria might lead to loss of revenue or client. The institutional structures and perception of software development in Nigeria as a whole rarely support the validation of local practices of work. Quality service is mostly considered when products or practices are imported (implying Western products are of high quality and their practices adaptable). The analysis of the process of project initiation, requirement gathering, and design thinking identifies how relations specific to the context of Nigeria are performed and enacted. From the localised perspective of practitioners, the abstraction that technological practices are cultural or politically neutral is falsified.

4.2.3 The Implication of Localised Practices - Educational and Designerly. This theme accounts for the implications of the more common pedagogical culture and practice in Nigeria that might have influenced the practice of designing and producing educational tools. Within the broader context of the Nigerian higher education sector, the data suggests that much relevance is given to the delivery of content, rather than how the technology can shift and impact the processes, activities and behaviours of different users. For example, three participants noted that the tools they develop and deploy are mostly concerned with the automation of certain instructional processes and activities rather than supporting an entire pedagogical experience (F1, F2, F7). The data also stressed the fundamental relevance of identifying implications from indigenous languages (more emphasis), local pedagogical approaches, industry and government policies, and the plurality of people's ways of knowing to the practices of undertaking project work. This would determine how project practices could be made to support and promote the consideration of local perspectives and the extent to which tools developed would reflect local circumstances and needs. Two Participants suggested that;

"there is the need to first understand how we study, how our young generation study and the generations to come, and then look at the technologies that will fit into those ways of learning by those learners" (F6).

"We are informed by the practice of other stakeholders because basically when it comes to learning, learning is a very sensitive aspect to the economy. Before you venture into that, you have to know what the standard is, what's organization are key and what policies are in place. For instance, with NERDC (National education research development council), they have their course curriculum for the student at different levels. I don't know if you have heard of TESSA (teacher education for Sub Saharan Africa), which is an organization that has already set standards as well. So, these are the kind of things or parameters we tend to look at and make sure that Yes, our content and platform are in line with the established standard, both national, regional and international" (F5).

It seems obvious that practitioners attempted to understand the plurality of learning cultures and preferences and used different pedagogical strategies at their disposal to transform the understanding into actionable insights that could inform design practices. This is relevant as one can begin to identify how a mismatch can be minimised, a continual collaboration between educational process and design practices supported, and the likelihood of developing pedagogical relevant and adaptable tools that can be scaled across different institutional context attained. A practical example is when the eLearning lead suggested that their eLearning system might have;

"a feel of local pedagogical needs attached to it. The person narrating the learning material has an African accent. So, the way she is 
interacting is actually the same way teachers teach in our institutions. This can be regarded as a local pedagogical consideration" (FF1).

This adds to the consideration of the implications of localised practices of designing and deploying educational tools, which could upscale the likelihood of user's seeing the need to adopt and use tools in their teaching and learning. It is also important to establish the thinking guiding the processes of designing and developing these technologies. What this theme emphasises is that the design of educational technologies without careful consideration of pedagogical culture and epistemological differences in design reasoning might lead to a project not meeting requirements or failing altogether. In the broad context of doing work translocally, the theme has raised a range of issues that necessitate a closer examination of how a range of practices and knowledge are to be used in appropriating and regenerating the practices of project work in a context such as Nigeria.

4.2.4 The Place of Effective Practices - Myths and Reality. The data identifies a range of ideas about the internal configuration and the external representation of software project work. This concerns how project processes and activities are organised and coordinated using a range of techniques and technologies; how requirements are transformed into design concepts, how to design scenarios are staged, and the strategies adopted in ensuring that a particular design and development methodology is adhered to. To some extent, these ideas determine whether what is considered effective 'best' practices are developed from the situated work of practitioners or whether it is merely about the appropriation of conventional 'best' practices of software development. From the analysis, all three companies attempt to follow traditional software development and well-known design approaches (meaning prescriptive maps and scripts assists in organising and coordinating project processes/activities) in their work. There is a general agreement with regards to what is considered effective 'best practices' of doing work - often referred to with the concept of what's 'out there' and what 'works'. As one participant noted:

"We learn from our own work, based on our experiences and with that of other people. We also attend workshops, submits and conferences like eLearning Africa. But the main issue is that when we attend such events, they usually tell you what's out there in the West. So, it is mostly about how we adopt things here" (F6).

What the account might suggest is that the localised practices of Edusoft projects are modelled through and aligned towards what the key players in the software development industry are doing. The 'out there' meant the practices that multinational companies develop and adhere to in their everyday work practice, whereas the 'in here' means the situated practices of practitioners in Nigeria. Such issues have been of concern in HCI $[4,35]$ as it has pointed to the power relations and oppositional binaries created by asymmetric differentiation of work practices. Practitioners assume that the key players have set the precedent for such practices to be considered 'effective'. The presumption is that when a framework works in various circumstance and organisational context, a community will rally behind it, and which might warrant it being considered as best practice (presuming that the realities and circumstance in developed and developing countries would be relationally similar).
Arguably, it is precisely in the process of adopting what's out there that neo-colonial and dominant relations between organisational constructs and management knowledge are normalised and universalised. A closer examination of the 'in here' within the framing of what 'works' might suggest the sensitivity and creativity of practitioners as they work with the understanding of the situated circumstance and realities of their work. In a participant's words for example:

"In ensuring quality of the platform as I said, we make sure we are designing with industry standard. We tend to look at what other stakeholders are doing, what makes the key players stand out, and how that applies to the Nigerian content, and then provide services that suite the Nigerian market, what is missing and how can I improve my product to be better in term of interfaces, user experience, user feel, make sure is mobile compatible, looking at speed optimization, so we put all this into consideration" (F5).

The reality is that they are not merely passive recipients or victim of appropriation but consider themselves as appropriators and co-creators of new ways of undertaking and accomplishing work. As dominant boundaries are relationally shattered through the lens of translocality, one might consider how the localised procedure of OKR might have assisted in providing an effective way of empowering and transforming the situated practices of practitioners in Edusoft projects. The OKR is an organizational aid box that assists in ensuring that the reasoning, processes, activities, and actions of each team are aligned to the company mission. Figure 1 and Figure 2 shows C1's OKR and the engineering department OKR. From the figure, one can appreciate the orderliness and timely progression of Edusoft project works, having achieved $70 \%$ and $84 \%$ overall scores. What the situated practice of the projects might suggest are ways in which effective practices are created, taken seriously, applied and sometimes ignored. Indeed, there is a myth of 'best practice' and there is also the reality of how local capacities and knowledge effectively inform the practice of doing project work. This might suggest where localised practices are more relevant and sustainable within the cultural context of Nigeria.

4.2.5 The Influence of Civic Structure and Organisational Contingencies. This theme holds a whole range of ideas relating to how software project processes and practices are influenced, shaped and impacted by the institutional structures and cultures in the Nigerian software industry. The culture of the industry is one that is driven by the assumptions of practitioners about design innovation and by the perception of the general public as to how the industry operates and what it can deliver. The cultures are shaped by the regulatory practices in Nigeria, the convention of the industry, the sort of diffusion and adoption strategies widely used, as well as the behavioural attitude of both producers and consumers of innovation. All participants admitted that as they work in a complex and emerging industry, and that the structures shaping the industry are mostly driven by Eurocentric ideals (in term of commercialisation, competitiveness, and cooperate strategizing). With the social, political, and infrastructural differences between those Western ideals that they model their practice on and the realities in Nigeria, it can be inferred that the mismatch would greatly influence and impact 
the possibilities of developing localised capabilities and practices. As it stands, the major issue is that, as Mavhunga rightly puts; "the dilemma of knowledge production in Africa centres on how its structures, practices and concepts come to be informalized while inbound European one's where rendered formal" [22 p. 10].

Regardless of the influence and impact of such a misguided and oversimplified assumption, all participant admitted that they had to devise ways of knowing and doing design that appeal to the cultural and institutional structures of the environment that they design for and deploys to - somewhat moving towards the relational and the pluriverse [12]. This is illustrated by a participant who suggested that project practices are aligned to deployable context, in term of cultures, religious affiliation, language (strong emphasis), local politics, and economic realities of users. The experience was with a school that requested that their academic transcript to be designed in the Arabic language mainly because it is an Islamic school. In his words:

"So, we had this client that insisted on having their report sheets in Arabic. We said OK fine we'll do so. But they should inform us on the percentage of parent that understand Arabic since the report sheets are meant for them to know the performance of their children. Then they realize that what they are asking for is feasible to us but not relevant to the immediate environment we find ourselves in" (F1).

"Looking at the culture, we now realize this language barrier in Nigeria and that there this tribalism associated with products.... if you have the platform in such a way that he/she is able to navigate in Hausa now it appeals to the user's emotion, creating that connection to the platform" (F5).

This is a typical account of what to expect from a client that is quite aware of the influence of the culture of the education and the context in which educational tools are to be deployed. There was also the recurrent emphasis on the issue of client pressure to produce tools within a shorter period of time, the perceived under-appreciation of software products by the general public, and the misguided understanding of software development to be more about open sources software (OSS). Although practitioner adopts an agile methodology to make work flexible and manageable, due to the constant pressure from clients and in an effort to react to those pressures as to retain clients in the competitive environment they work, some critical processes of work are neglected as a consequence of being overly flexible and reactive. This is emphasised by the engineering team lead who suggested that;

"The main thing that is affecting our processes is the manner in which projects are coming in and the duration of projects. There is no time to do quality assurance. The main priority is trying to meet the deadline as we are always on a rush. They transfer the pressure from the client to management and onto the team. This I think is the main thing that affects the standardising of processes or implementing the western way of doing software projects in Nigeria" (FF6).

As agile might not account for such contingencies, what stands out is the indigeneity of practitioners in understanding such issues and in devising means towards minimising or absorbing their effect in their everyday work. It is the cultural agility of Edusoft project processes and activities that could minimise following neo-colonial rules while emphasizing the creation of new rules of work that are situated, transitional and transnational. In essence, what the theme points to is that these issues are not a simple a matter of understanding and conceptualising local experiences but also global one's within the framing of cosmopolitan localism.

4.2.6 The 'Wickedness' of Distributed and Collaborative Project Work. The ideas contained in the themes above connect broadly to generic consideration of how the conflicting issues created in work while attempting to adopt best practices might provide an avenue for recognising and harnessing localised practices. This is considered in relation to the sort of difficulties and issues designers and developers face in their everyday work due to attempting to follow conventions (regarded as dominant and prescriptive rules). We are particularly interested in the 'mess' rule-following might create as the consequences of the inevitable nature of collaborative and distributed work. I refer to these issues as 'wicked' problems mainly because we are not after finding a correct path but pointing to some neglected ideas of how to better understand the problem of design and development in a context where cultures are more apparent. To give an example of how designers and developers attempt doing distributed and collaborative is to account for how agility is actually done. The data suggested that practitioners engage in creating personas and use case diagrams, brainstorming ideas, have design and development sprint, conducting wire framings, develop user flow testing and evaluation, conduct design assumption test scenarios, design high fidelity mock-ups, develop design and development backlogs, develop content prototypes, and develop scrum as they allow quicker development, testing and quality assurance. But most participants admitted that they do not follow the classic approach to agile scrum methodology (EVF, F1, F6, FF2, FF5), but rather a 'partial agile scrum methodology' (FF3, FF6). This is supported by a participant who suggested that;

\section{"In this company, we are not doing the complete process of agile project management.... due to the nature of the way projects are coming, clients are always in a hurry, so we have to take it's as it comes. If not, they will give it to a different company. We just do things and we just call it agile project management as we use Agile fira board..... We are using the tools but in a semi-structured way. We are just combining different tools and approaches" (FF6).}

What their perspective suggests is that although they have attempted to do agility, due to the influence of civic structures and organisational contingencies narrated above, the level of agility is greatly hindered. Doing 'partial' agility was warranted by a range of factors, including the nature of projects coming, the way requirements evolve and get contextualised, the limitations of time to follow agile principles, the contextual pressure from clients, and the temporal mismatch between specification and expectances. The situated practice of Edusoft projects is that work is an iterative and continual process of ideation and exploration of concepts - somewhat an opportunistic design process. Although different structural 
strategies are adopted in the process of opportunistic design, deviation is possible and often inevitable. During the iterative processes, new requirements are discovered which warrant developing immediate solution before the next iteration. Another aspect of the wickedness of agility to localised practice is about how flexible one can be? To what extent one can negotiate? And to what extent one is willing to trade best practice for a business opportunity? As it appears, practitioners often become bureaucrat, lobbyist, and diplomats in order to secure project opportunities. With the sort of difficulties practitioners face while attempting to transition between different rules, it seems obvious that their situated practices are greatly affected as a result. This calls for a pluriversal approach to design thinking where the rules for interpretation and representation are reoriented towards the social context and culture one designs from, for, and with.

From the six themes identified and discussed, it is evident that doing agility is not merely a matter of adhering to the agile principles but more about how applicable, appropriate, and sustainable conventional practices of doing and accomplishing project work are. The themes might suggest how a range of approaches, constructs, concepts, and practices are important in shaping the practice of doing project work transitionally and translocally. The themes have thus raised and point to a range of ideas and issues that might suggest how agility can either be an orderly or a messy alternative for keeping work on track and as a totality. In essence, the analysis accounts for how local capacities and everyday practice/knowledge appropriately inform project work, which thereby shows where conventions are applicable and irrelevant and where indigenous sensitivities are more relevant and sustainable.

\subsection{The Possibilities of Developing Relational Practices through 'Remixing' and 'Playfulness'}

Accounting for and representing lived experiences of design work has been a major issue in understanding the materiality of transnational HCI. How then can we account for and represent the situated practice of Edusoft projects within a transnational space of design where there is an equal and an uneven relation of power between where practices enacted and where they get appropriated? To answer to that, I consider how the notion of transitionality [12] and translocality [6] of design practices can allow appropriating, regenerating and redistributing the practice of software practitioners in Nigeria. Transition, as a critical approach to designing for the pluriversal, emphasizes a relational cosmology and a multivalence ontology for knowing and doing design innovation [12]. Translocality focuses attention on how a range of localized strategies are enacted, performed and nourished in the agility of practitioners. It is presumed that doing 'partial agility' would allow reframing dominant practices that have continuously informed the design and development of technology in developing countries - as a precursor for postcolonial and decolonial approach to design thinking.

As the practices shaping the understanding and designing of technologies travel across boundaries, they encounter new conditions and challenges. It is through such exchanges that new meanings of appropriation are identified [36]. The emphasis is on how the continual reorientation of the culture of adoption and appropriation can adequately represent the localised practices of software practitioners in Nigeria. As the end product of Edusoft project are deployed to a wide range of user's, how then does the regeneration and redistribution of localised practices and knowledge takes places (or can take place)? Taking a translocal account of the practices of Edusoft, it becomes evident that the reorientation of the situated practice of work is not merely about showing how inbound strategies are translated for local circumstances, but more about how they get and transformed into new idioms for outbound transplantation [36]. It is in this transient space of continual engagement that the mythical abstraction of innovative practices from the global South can be interrogated, and the possibility of reframing from such myth initiated so that a pathway for understanding the creativity in 'adaptability' and 'strategic change' can be identified.

Within the framing of transitionality, the culture of 'remixing' [21] and 'playfulness' [2] between consumed and produced practices provide a way of regeneration and redistribution within and across existing and emerging boundaries. To outline the implication of remixing in constitutively producing alternative cultural practice is to consider how it has been adopted and extended in the literature as a pathway for collaborative learning. Simply, remixing is a form of fairly combining existing concepts and technologies to derive new ones [21]. It is often referred to as a productive 're-interpretive' process, a continual activity of 'mashup' and 'co-creation', and as a 'democratise' path of 'peer production' $[9,16]$. Some of the sociotechnological factors that drive remixing of project processes and activities are shared communal values, the multidirectional collaboration between initiators and remixers, and the emphasis on relationship creation than the reproduction of practices. Although remixing can either be extended, selective or reflexive, the major issue raised concerning its structures relate to its generativity, originality, or quality $[7,9]$. How then can the adoption and appropriation (through remixing culture) of monopolized organisational practices by software practitioners in Nigeria bring about the regeneration and the redistribution of their localised practice of project work?

The practice of following normative rules (maps and scripts) in doing and accomplishing Edusoft project work is not primarily about how conventional practices get interpreted and made useful, but more about the local rules that inform how they are taken seriously or ignored altogether. Therefore, the remixing of those maps and scripts through local rules could be extended as reusable maps and scripts specific to one's culture and context of work. This presents the creation and following of new rules (through a reflexive culture where rules are not a derivate of the convention but an extension of them) to be an emerging practice that is defined by socio-cultural norms that are situated and generative. A practical example of the remixing culture adopted in $\mathrm{C} 1$ is the suggestion that;

"We follow the standard of agile scrum not strictly but by looking at the environment we work in.....We have to apply it the way we apply it if you want things done. In Nigeria, few companies like Andela, eHealth Africa and Paystack adopt modern practices, some don't have advance standards. ... this gives the wrong impression 
that an application can be fully rolled out in a month." (FF5).

Such an account might suggest the dichotomy between where rules get initiated and where they get used and extended. It also shows how the creation and following of new rules might allow equalizing the power relations that already existed in transnational design spaces. In minimizing the privileging of specific practice over others in such a space of regeneration and redistribution, I consider how the 'playfulness' of cultural possibilities in software projects can provide resourceful way of presenting and representing multiple experiences without re-inscribing the dichotomy of the local-global [2]. To demonstrate the playfulness of practitioners for example, the eLearning lead suggested that:

\begin{abstract}
"I worked with a guy sometime back and he was trying to design a music mobile app for Africans. In trying to brand the application, he travelled to some countries in African to see what's unique about the context. At the end of the day, he was able to pick colours from animals that common in a different part of the continent. I think at the end of the day he named the app monkey music" (FF1, EVF).
\end{abstract}

So, the playfulness here is mainly about the cultural practice of considering pluriversal ways of design reasoning as the vintage point of undoing and unlearning dominant design practices [11]. Practitioners play off between adopting and neglecting universalized knowledge of design and development, thereby demystifying dominant practices of knowledge production as the playful cocreation would show what was created, expressed, lost, and gained in the continual exchange and negation between inbound and outbound strategies. The consideration of remixing and playfulness within the context of Edusoft projects s provide a relational way of participating in transnational relations of design and of representing their unintended and unacknowledged consequences.

\section{DISCUSSIONS AND CONCLUSION}

This study involves a sensitization to and an evaluation of the everyday processes and activities of distributed and collaborative software project work in Nigeria. The interest is in what actors are doing, actual software project work, and not some 'idealised' and 'sociologically interesting' or 'theoretically relevant' work [30]. What the case provides is a bird's eye view of the practices of producing software in the multi-cultural context of Nigeria. Significantly, the study notes the influence of civic structures, educational culture and social context of use on judgements of the appropriateness of certain organisational practices and knowledge for distributed and co-operative project work. The findings presented provide typical reflections of member's understanding of specific work practices, and not some idealised narrative of the ordering of project work, and more generally social life in Nigeria.

As earlier indicated, there appears to be an acknowledgement among participants of the importance of using design and engineering approaches that are flexible, iterative and adaptive to the contingencies of the environment and evolving user requirements. Due to the inevitable situated nature of work, the over-reliance of practitioners on Western conventions might have ultimately led to unnecessary difficulties in their work. Generally speaking, the findings support the assumption that what might stand as conventional best practice are social orders and prescriptions that are universalised through the matrix of power relations, which ultimately needed to be translocally decolonised. In addition, the data suggested that the practitioner's play-off between attempting to adhere to standard practices while also being flexible to their everyday working conditions. As such, practitioners are sometimes working together or sometimes against each other in order to ensure that agility is maintained; ensuring that projects works are carefully structured and ordered and not simply carried out at will. While doing so might lead to tension in work, the assumption is that practising 'cultural agility' would allow a balance between the need for standardization and flexibility [37]. The balancing can be considered as working with what is either best and doable (either through the practice of partial agile, cultural agile, or blind adoption of the practices of key players out there).

From the continual process of balancing between best-doable practices, it can be inferred that practitioners develop and deploy products that meet to the needs of the Nigerian educational sector largely because of their collective understanding of their work culture (plurality) and the context of deployment (pluriversal). In a participant's worlds:
"We build our system from scratch, even when we might not fully understand the theory and the practice of the environment. Another thing is, in this part of the world, software is not really valued highly. Some schools feel such platforms are luxury when we have more press- ing needs like lack of infrastructure. So, in order to get accepted, we hath to move overboard. Some of our col- leagues in diaspora think we have been over-engineering and have been underutilized in several instances" (F1).

This is exactly what the localisation of project work might signal, that technological innovation happens in/from Africa like any other advanced nation. What standout, however, are context-specific attributes that differentiate different challenges and opportunities in the Nigeria software industry, like that of the broader context of Africa. Ultimately, the localised practices of the creators of Mpesa in Kenya will be different from that of Paystack in Nigeria, for example. There might be significant differences in context and culture, but what I am trying to emphasis is how the localisation of work practice can bring about the development of a unified language for the analysis and the documentation of organisational practices and knowledge within the context of Nigeria, and Africa at large. It is within the unification that the language affords that the utility of conventional and localised practice can be made available for distribution and appropriation across contested boundaries.

As the practice of project work in Nigeria is shaped by uneven and globalist assumptions and principles of design, the analysis raised and offered a range of ideas that point to how the praxis of design can be endogenized for the betterment of those that were marginalised and those doing the marginalisation [33]. Although practitioners in the three companies have acknowledged the differences in the conditions that they work and of those that they are blindly following, they fail to appreciate that their processes of devising local logicalities of accomplishing work are more productive and important constructs for informing and shaping future work 
than any adopted practices 'out there'. Whilst I do not suggest that the adoption of stereotypical western approaches is wrong or detrimental to the decolonisation of localised practices and knowledge, what I point to is that possibilities of constitutively appropriating and regenerating situated practices of practitioners in Nigeria, translocally or largely a transition of cosmopolitan localism [12].

In understanding software project work in the multicultural context of Nigeria, the case study emphasises findings widely reported in the literature concerning the implication of doing agility in collaborative and distributed project work [37, 39], and specific to developing countries [20]. It also identifiers similar trends to [24, 26, 28] findings that the Nigerian software industry work practice is more concerned with automating manual work than of creating a whole chain of system. Findings also point to lack of awareness of end user's positionality in shaping and subsequently ensuring acceptability of deployed end products [10,20], the rampant adoption of development methodologies mainly because that's what the 'key players' in the industry are adopting [28], and the likelihood of abandoning situated practice for Western conventions [26]. There was an emphasis on the informality of project processes and activities, the over-politicisation of design decisions, and the trade-off of profitability over nurturing local capabilities, explicating earlier findings by [39]. These factors can be relatively attributed to the political instabilities and economic difficulties practitioners face in their work, which characterises the complexities and volatility of the Nigerian software industry.

Apart from the implications of the themes developed, findings point towards the idea that designing and producing software using agile is an expensive gamble at the crossroad of one's organisational practice of doing agility and of one's personal productivity and professional development [5, 37]. As it stands, practitioners are innovating for survival and from below the radar, which might suggest the 'darker side' of project work, and in particular agility $[5,6]$. It also shows the imbalance between standardization of practices (through following of rules) and the needed flexibility that agile espouses - restating some of the classic problems of CSCW as emphasised by [37]. This might suggest that project work is not entirely an engineering phenomenon (the focus has shifted to software projects), but also a thread of socio-cultural, economic, political, and material concerns that are ultimately determined by power relations. It also emphasizes the classic understanding that software development is an iterative process of reasoning, decision making, reflection, negotiation, and validation, which ought to be approach and contextualise as such.

Within the framing of translocality, the insights that came out of the consideration of the concept of 'remixing' and 'playfulness' are meant to reframe widely held assumptions about design innovation in Africa. By showing how Edusoft projects practitioners innovate within the transient spaces accorded by transitionality, I have attempted to indicate the politics and materiality of innovating from below the radar - somewhat thinking and making from the borders. The orderly and messy features of distributed and collaborative work evoke by the case are to serve as (or can serve as) both political (in the sense of identifying and focusing on neglected blind spots) and analytical strategies for critiquing and unlearning dominant and neo-colonial practices of design innovation in non-western context [38].
Within the wider context of the literature, accounting for the lived experiences of doing Edusoft projects has moved towards showing the myth of design innovation in/from Africa - the localised practices of practitioners and their circular processes of diffusion, appropriation, translation, and contestation are innovative $[22,40]$. The practitioners I worked with rely on their experiences and the understanding of the conditions and circumstances of the present in identifying and developing new ways of doing work that would further inform their present and future practices. Practitioners reflexively think and act along the learning paths accorded by their shared experiences and knowledge of the people that they design with/for and social context they deploy to, not some idealised and grand narrative of reacting to dominant practices and standards. The juxtaposition of different procedures, strategies and technologies available to them productively open up new possibilities outside current binaries of what is 'best practice' and 'doable practice'; thereby outlining propositional offerings for making meanings and senses of the approaches widely considered for framing and ordering project work. To end on Escobar's note, transitional and translocal design can be considered as a "means to think about, and to contribute to the transition from a hegemony of modernity one-world ontology to a pluriverse of socio-natural configuration, in this context, design for the pluriverse becomes a tool for reimagining the reconstructing the local world" [12, p.4].

\section{ACKNOWLEDGMENTS}

I am a Nigerian interested in postcolonial approaches to developing indigenous pedagogies and the design of indigenous technologies that embodies them. It is presumed that accounting for one's positionality might situate the researcher and the research participant as subjects of interactivity in knowledge production (I thank a reviewer for bringing my attention to this - a matter of greater importance to standpoint theory which I subscribe to). I am indebted to my supervisors, Mark Rouncefield and Philip Benachour for the usual advice to 'put more data', and 'tighten up the argument a bit'. I am also grateful to the participants that inform this project and thank the anonymous reviewers for insightful comments on early draft of this paper. This paper is part of a $\mathrm{PhD}$ research funded by the Petroleum Technology Development Fund (PTDF), Nigeria.

\section{REFERENCES}

[1] Adamu, M. S. (2020, April). Adopting an African Standpoint in HCI4D: A Provocation. In Extended Abstracts of the $2020 \mathrm{CHI}$ Conference on Human Factors in Computing Systems (pp. 1-8).

[2] Anderson, R. J. (1994). Representations and requirements: the value of ethnography in system design. Human-computer interaction, 9(2), 151-182.

[3] Avgerou, C. (2008). Information systems in developing countries: a critical research review. Journal of Information Technology, 23(3), 133-146.

[4] Avle, S., \& Lindtner, S. (2016, May). Design (ing)' Here' and 'There' Tech Entrepreneurs, Global Markets, and Reflexivity in Design Processes. In Proceedings of the 2016 CHI Conference on Human Factors in Computing Systems (pp. 22332245).

[5] Bjørn, P. (2019). Dark Agile: Perceiving People As Assets, Not Humans. In Rethinking Productivity in Software Engineering (pp. 125-134). Apress, Berkeley, CA.

[6] Bjørn, P., Søderberg, A. M., \& Krishna, S. (2019). Translocality in global software development: The dark side of global agile. Human-Computer Interaction, 34 (2), 174-203.

[7] Cheliotis, G., Hu, N., Yew, J., \& Huang, J. (2014, February). The antecedents of remix. In Proceedings of the 17th ACM conference on Computer supported cooperative work \& social computing (pp. 1011-1022). 
[8] Crabtree, A., Tolmie, P., \& Rouncefield, M. (2013). "How Many Bloody Examples Do You Want?" Fieldwork and Generalisation. In ECSCW 2013: Proceedings of the 13th European Conference on Computer Supported Cooperative Work, 21-25 September 2013, Paphos, Cyprus (pp. 1-20). Springer, London.

[9] Dasgupta, S., Hale, W., Monroy-Hernández, A., \& Hill, B. M. (2016, February) Remixing as a pathway to computational thinking. In Proceedings of the 19th ACM Conference on Computer-Supported Cooperative Work \& Social Computing (pp. 1438-1449)

[10] Dingsøyr, T., Nerur, S., Balijepally, V., \& Moe, N. B. (2012). A decade of agile methodologies. Journal of Systems and Software, 85(6), 1213-1221.

[11] Dourish, P., \& Bell, G. (2011). Divining a digital future: Mess and mythology in ubiquitous computing. MIT Press

[12] Escobar, A. (2018). Designs for the pluriverse: Radical interdependence, autonomy, and the making of worlds. Duke University Press.

[13] Glaser, B. G., \& Strauss, A. L. (2017). Discovery of grounded theory: Strategies for qualitative research. Routledge.

[14] Halverson, C. A. (2002). Activity theory and distributed cognition: Or what does CSCW need to DO with theories? Computer Supported Cooperative Work (CSCW), 11 (1-2), 243-267.

[15] Heeks, R. (2002). Information systems and developing countries: Failure, success, and local improvisations. The information society, 18(2), 101-112.

[16] Hill, B. M., \& Monroy-Hernández, A. (2013). The remixing dilemma: The tradeoff between generativity and originality. American Behavioral Scientist, 57(5), 643-663.

[17] Irani, L., Vertesi, J., Dourish, P., Philip, K., \& Grinter, R. E. (2010, April). Postcolonial computing: a lens on design and development. In Proceedings of the SIGCHI conference on human factors in computing systems (pp. 1311-1320).

[18] Kinnunen, H., \& Luoma, E. (2018). Towards measuring the agility of software business. In Proceedings of the Annual Hawaii International Conference on System Sciences; University of Hawai'i at Manoa.

[19] Korpela, M., Soriyan, H. A., Olufokunbi, K. C., \& Mursu, A. (2017). Made-in Nigeria systems development methodologies: An action research project in the health sector. In Information Technology in Context: Studies from the perspective of developing countries (pp. 134-152). Routledge.

[20] Kunda, D., Mulenga, M., Sinyinda, M., \& Chama, V. (2018). Challenges of Agile Development and Implementation in a Developing Country: A Zambia Case Study. JCS, 14(5), 585-600.

[21] Lessig, L. (2008). Remix: Making art and commerce thrive in the hybrid economy. Penguin.

[22] Mavhunga, C. (2017). What Do Science, Technology, and Innovation Mean from Africa? The MIT Press.

[23] Millen, D. R. (2000, August). Rapid ethnography: time deepening strategies for $\mathrm{HCI}$ field research. In Proceedings of the 3rd conference on Designing interactive systems: processes, practices, methods, and techniques (pp. 280-286)

[24] Mursu, A., Soriyan, H. A., \& Korpela, M. (2018). Risky business: A case study on information systems development in Nigeria. The Digital Challenge: Information Technology in the Development Context: Information Technology in the
Development Context.

[25] Nicholson, B., \& Sahay, S. (2001). Some political and cultural issues in the globalization of software development: case experience from Britain and India. Information and Organization, 11(1), 25-43

[26] Ogunyemi, A. A., Lamas, D., Adagunodo, E. R., Loizides, F., \& Da Rosa, I. B. (2016). Theory, practice and policy: an inquiry into the uptake of HCI practices in the software industry of a developing country. International Journal of HumanComputer Interaction, 32 (9), 665-681.

[27] Ogunyemi, A., Lamas, D., \& Eze, E. (2018). Exploring the State of Human-centred Design Practice in Software Development Companies: A Cross-Case Analysis of Three Nigerian Software Companies. Interacting with Computers, 30 (5), 444-467

[28] Ogunyemi, A., Lamas, D., \& Stage, J. (2016, November). Do Contexts Make A Difference? Software Practitioners' Perspectives on HCI Practice and Integration to Software Engineering Processes. In Proceedings of the First African Conference on Human Computer Interaction (pp. 92-103).

[29] Passos, C., Cruzes, D. S., Dybå, T., \& Mendonça, M. (2012, September). Challenges of applying ethnography to study software practices. In Proceedings of the 2012 ACM-IEEE International Symposium on Empirical Software Engineering and Measurement (pp. 9-18). IEEE.

[30] Randall, D., \& Rouncefield, M. (2018). Ethnographic Approach to Design. The Wiley Handbook of Human-Computer Interaction Volume, 125

[31] Rönkkö, K., Dittrich, Y., \& Randall, D. (2005). When plans do not work out: How plans are used in software development projects. Computer Supported Cooperative Work (CSCW), 14(5), 433-468.

[32] Schmidt, K. (1997, November). Of maps and scripts-the status of formal constructs in cooperative work. In Proceedings of the international ACM SIGGROUP conference on Supporting group work: the integration challenge (pp. 138-147).

[33] Schultz, T., Abdulla, D., Ansari, A., Canl, E., Keshavarz, M., Kiem, M., ... \& JS Vieira de Oliveira, P. (2018). What is at stake with decolonizing design? A Roundtable. Design and Culture, 10 (1), 81-101.

[34] Shklovski, I., Vertesi, J., \& Lindtner, S. (2014). Introduction to this special issue on transnational HCI. Human-Computer Interaction, 29(1), 1-21.

[35] Taylor, A. S. (2011, May). Out there. In Proceedings of the SIGCHI Conference on Human Factors in Computing Systems (pp. 685-694). ACM.

[36] Tchounikine, P. (2017). Designing for appropriation: A theoretical account. Human-Computer Interaction, 32(4), 155-195.

[37] Tendedez, H., Ferrario, M. A. F. C., \& Whittle, J. N. D. (2018). Software Development and CSCW: Standardization and Flexibility in Large-Scale Agile Development. Proceedings of the ACM on Human-Computer Interaction-CSCW, 2 (CSCW).

[38] Tunstall, E. (2013). Decolonizing design innovation: Design anthropology, critical anthropology, and indigenous knowledge. Design anthropology: theory and practice, 232-250.

[39] Vallon, R., da Silva Estacio, B. J., Prikladnicki, R., \& Grechenig, T. (2018). Systematic literature review on agile practices in global software development. Information and Software Technology, 96, 161-180.

[40] Williams, L. D., \& Woodson, T. S. (2012). The future of innovation studies in less economically developed countries. Minerva, 50(2), 221-237. 


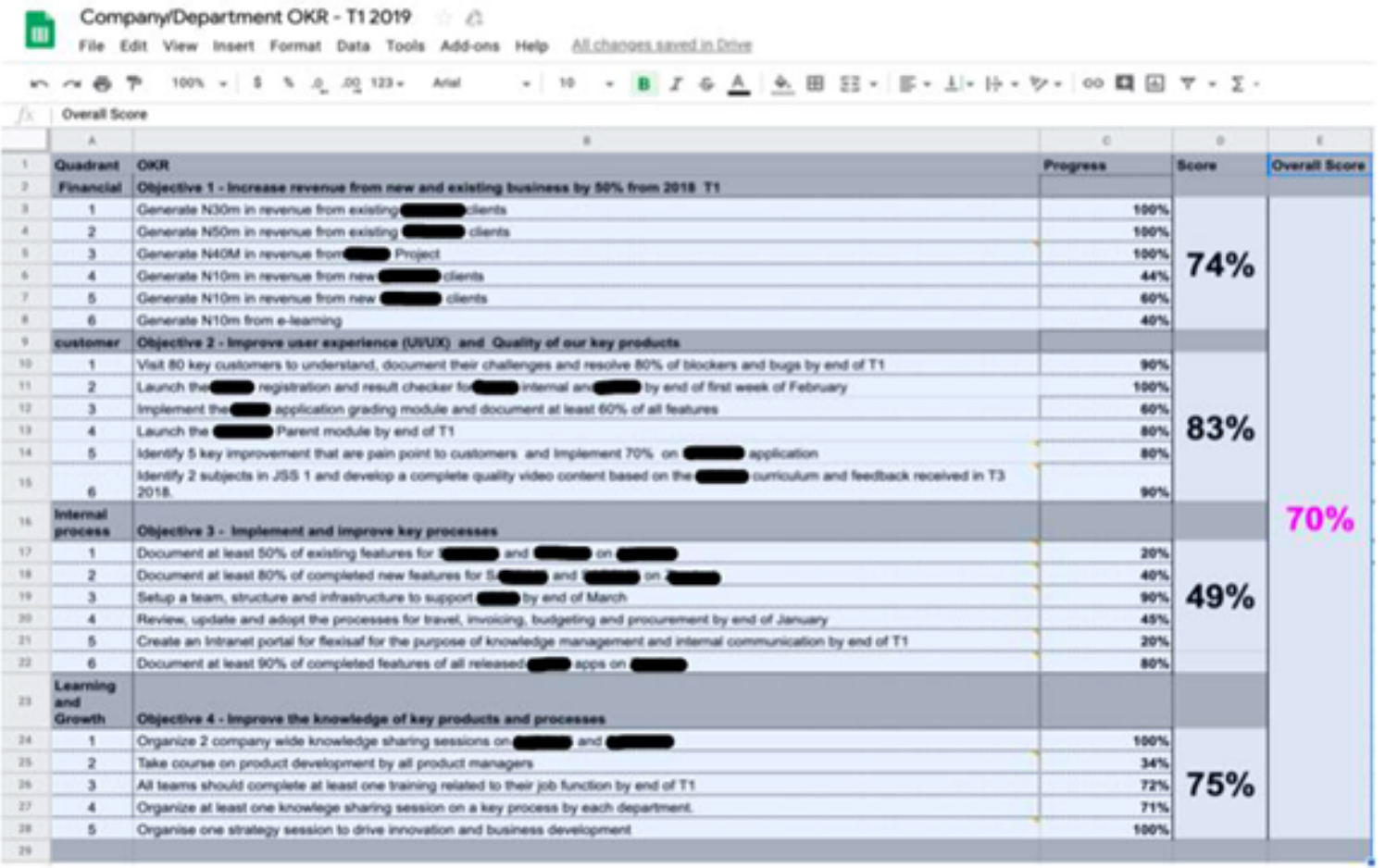

Figure 1: Edusoft Company Objective and Key Result indicators

Company/Department OKR - T1 2019

File Edit View Insert Format Data Tools Add-ons Help All changess saved in Drine

\begin{tabular}{|c|c|c|c|c|c|}
\hline$\frac{n}{\sqrt{x}}$ & $\begin{array}{l}x \bar{\nabla} \quad 100 x \\
\text { Quadrant }\end{array}$ & 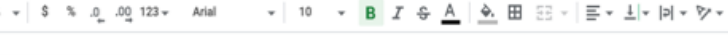 & $\infty \mathbf{q}$ & 回 $7 \cdot \Sigma \cdot$ & \\
\hline & A & B & c & D & $\mathrm{E}$ \\
\hline 1 & Quadrant & OKR & Progress : 5 & score & Overall Score \\
\hline 2 & Quality & Objective 1 - Improve avallability and quality of Key Features & & & \\
\hline 3 & & 1 1. Investigate what led to downtime and ootimise to prevent feature reoccurence by end of $\mathrm{TI}$ & 90\%: & \multirow{3}{*}{$87 \%$} & \multirow{13}{*}{$84 \%$} \\
\hline 4 & & 2. Fix known bugs on report queve and automate spinning of new senvers when more roport tratec is detected by end of march & 75\%: & & \\
\hline s & & $\begin{array}{l}\text { Remove } 100 \% \text { of unused code and refactor } 80 \% \text { of rodundant oode in al mngrs related to student reportsheet and accounts on } \\
\text { 3. Mand } 100 \% \text { on } \\
\text { Parent by end of March }\end{array}$ & 95\% & & \\
\hline 6 & unux & Objoctive 2 - Improve user experience (UIUX) on Key Feastures & & & \\
\hline 7 & & 1) Resoche (80\% of blockers and bugs) challonges collocted and documentiod from 80 key customer visits by ond of T1 & 90\% & \multirow{3}{*}{$72 \%$} & \\
\hline . & & 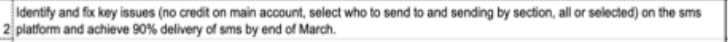 & $75 \%$ & & \\
\hline • & & 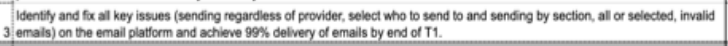 & 50\% & & \\
\hline 10 & Parents Satistaction & Objective 3 - Launch Parents Web Module and Parents Moblie App by end of march & & & \\
\hline " & & 1 B Bulild an activity dashiboard to increase parent engagement by end of march & $100 \%$ & \multirow{3}{*}{$80 \%$} & \\
\hline 12 & & 3 Make al pages responsive for different screen sizes for mobile and tabilet users by end of march & 90\%: & & \\
\hline 1 & & 4 Build a parent mobilo app with at least 2 features (result, tees and mobile notfication) by end of T1 & 50\% & & \\
\hline${ }^{4}$ & Capacity Bullding & Objective 4 - Improve the knowledge of key products and processes & & & \\
\hline 15 & & 1 Organize a compary wide knowledge sharing sestion on by mid-Aparl & $100 \%$ & $98 \%$ & \\
\hline$\frac{16}{17}$ & & 2 Al engineers must tako a course on Tosting rolated to their tochnology by end of T1 & $96 \times$ & & \\
\hline in & & & & & \\
\hline 19 & Engineers & Course Type & Propress & & \\
\hline 20 & & Testing & $100 \%$ & & \\
\hline$n$ & & Tosting & ss5: & & \\
\hline$n$ & & Testing & 100\% & & \\
\hline z) & & Tosting & $100 \mathrm{x}$ & & \\
\hline
\end{tabular}

Figure 2: Edusoft project development team OKR indicators 


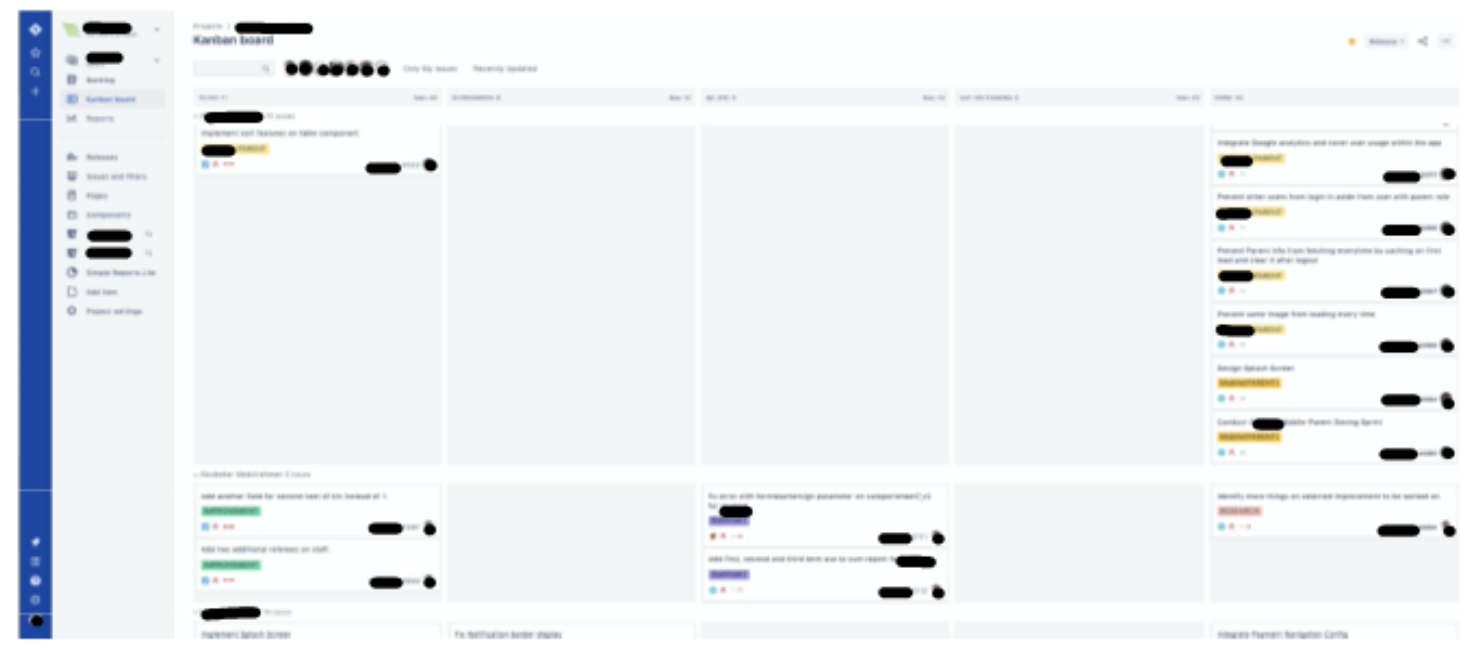

Figure 3: Nemis Scrum board for everyday project work

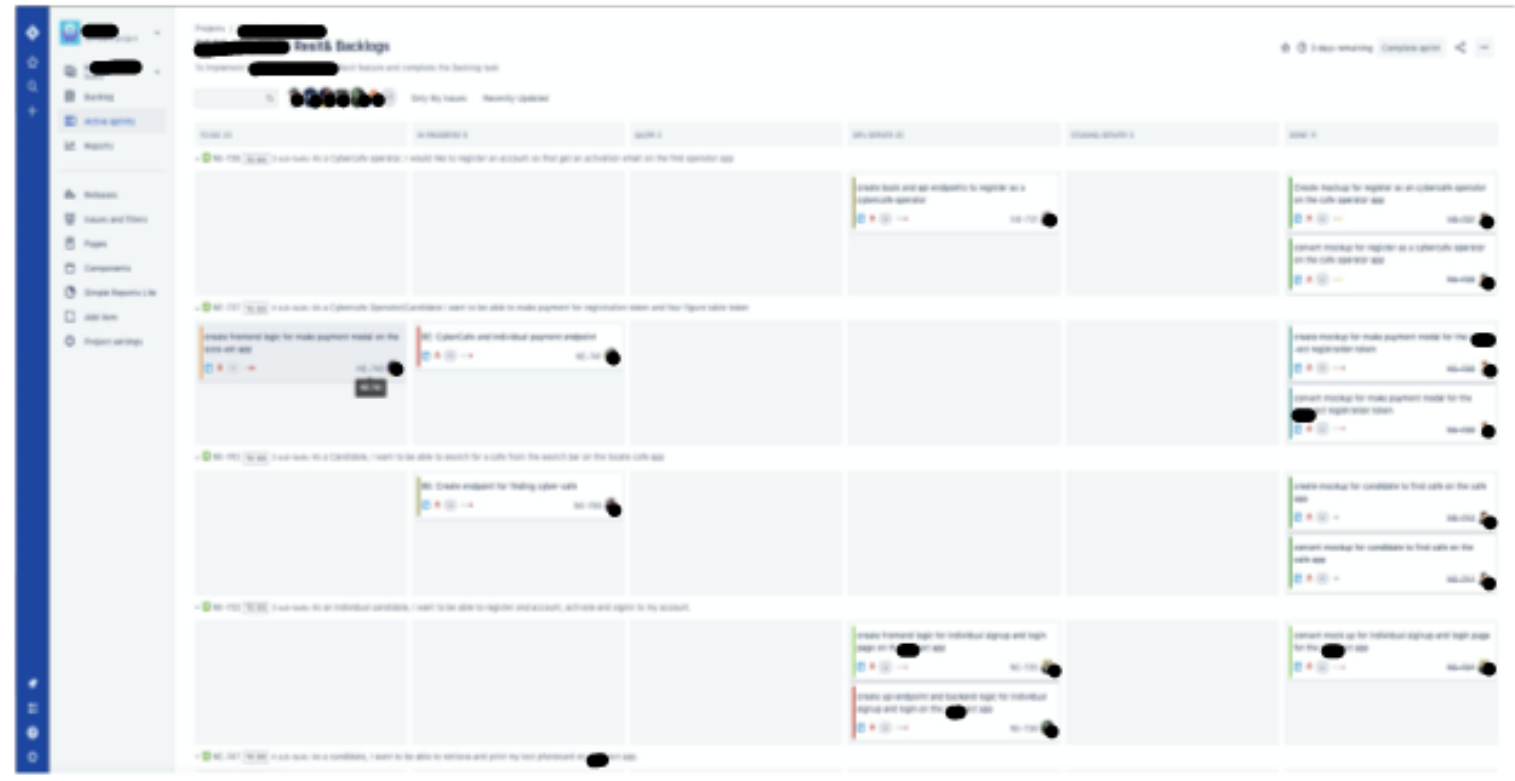

Figure 4: Kanban board for smaller projects 